\title{
An Ant Colony Optimization Algorithm for Microwave Corrugated Filters Design
}

\author{
Ivan A. Mantilla-Gaviria, ${ }^{1}$ Alejandro Díaz-Morcillo, ${ }^{2}$ and Juan V. Balbastre-Tejedor ${ }^{1}$ \\ ${ }^{1}$ Instituto ITACA, Universidad Politécnica de Valencia, Camino de Vera S/N, Edificio 8G, Acceso B, Planta 3, 46022 Valencia, Spain \\ ${ }^{2}$ Departamento de Tecnologías de la Información y las Comunicaciones, Universidad Politécnica de Cartagena, Cartagena, \\ Plaza del Hospital 1, 30202 Cartagena, Spain
}

Correspondence should be addressed to Ivan A. Mantilla-Gaviria; iamantillagaviria@gmail.com

Received 29 April 2013; Accepted 5 June 2013

Academic Editor: André Nicolet

Copyright (C) 2013 Ivan A. Mantilla-Gaviria et al. This is an open access article distributed under the Creative Commons Attribution License, which permits unrestricted use, distribution, and reproduction in any medium, provided the original work is properly cited.

\begin{abstract}
A practical and useful application of the Ant Colony Optimization (ACO) method for microwave corrugated filter design is shown. The classical, general purpose ACO method is adapted to deal with the microwave filter design problem. The design strategy used in this paper is an iterative procedure based on the use of an optimization method along with an electromagnetic simulator. The designs of high-pass and band-pass microwave rectangular waveguide filters working in the C-band and X-band, respectively, for communication applications, are shown. The average convergence performance of the ACO method is characterized by means of Monte Carlo simulations and compared with that obtained with the well-known Genetic Algorithm (GA). The overall performance, for the simulations presented herein, of the ACO is found to be better than that of the GA.
\end{abstract}

\section{Introduction}

Several kinds of microwave filter design procedures have been proposed in the literature along the time. Almost all of them can be grouped into two categories; one of them includes those methods consisting of two stages; synthesis and optimization [1]. In the synthesis stage, techniques based on lumped elements and equivalences between these and physical models are used. The other category includes those approaches which are only based on the use of optimization techniques along with an electromagnetic simulator [1-4]. The procedure considered herein lays on the latter category.

As described in [4], it is often necessary to implement two kinds of optimization: first, a global optimization method is used to find a roughly good solution and then local optimization is carried out to improve the previous solution. For the global optimization phase it is common to use some heuristic strategies [2-6] while for local optimization deterministic methods like the simplex method [7] or gradient based algorithms (e.g., the Broyden-Fletcher-GoldfarbShanno applied in [4]) are usually employed.
The Ant Colony Optimization (ACO) is a kind of metaheuristic optimization method developed by Dorigo $[8,9]$. The ACO is based on the communication system used by ants when they are looking for food sources. This system is an indirect system based on the modification of the physical environment by means of the segregation of a substance called pheromone $[8,9]$. It has been widely applied to different engineering problems. However, in the electromagnetic community it has been mainly applied to the synthesis of antenna arrays [10-15], whilst only few applications have been found for the design of microwave filters. Particularly, in [16], the design, optimization and experimental validation of a microwave filter with Split Ring Resonators (SRR) is shown. In that work the parameters of the SRR (four parameters) were optimized by means of a particular ACO algorithm (described in [12]), used more as a local search algorithm than a global search one.

The aim of this work is to apply this novel and robust metaheuristic optimization technique, the ACO, to the design of microwave corrugated filters based on the strategy commented above. Herein, the ACO method is only used for global optimization phase and the results are compared with 
those obtained with the well-established Genetic Algorithm (GA) method. The paper is organized as follows. In Section 2, a short summary of the ACO method is presented. Then, the application of the ACO method to the $\mathrm{H}$-plane microwave corrugated filters is shown in Section 3, where the bases of the method are outlined and the modifications implemented in the original algorithm, described in [17], are explained in some detail. Finally, numerical examples are shown in Section 4 and the results are compared with those obtained using the well-known GA method.

\section{Ant Colony Optimization Algorithm}

The ACO method is based on the communication system used by ants when they are looking for food sources. This system is an indirect system that is based on the modification of the physical environment by means of segregation of a substance called pheromone.

When a bifurcation of the path appears, ants choose one of these with a probabilistic rule biased by the amount of pheromone. This probability is directly proportional to the level of pheromone, that is, the higher pheromone level in a path the higher probability to be chosen. In Figure 1 the foraging process is described.

Figure 1 shows the ant colony nest (it is assumed a number of eight ants for this example), a source food, and two possible paths between them. The colour intensity represents the pheromone level for every path. Moreover, it is assumed that the shortest path is the path $\mathrm{A}$ and it has a travel time of one time unit, whilst the path $B$ is the longest one with a travel time of two time units. For $t=0$, the ants are in the nest and go out to search for food. The initial selection of one path is random. It is assumed that half of the colony chooses the path $\mathrm{A}$ and the remaining one the path $\mathrm{B}$. For $t=1$, the ants which travelled on the path A are in the food source and they have segregated an amount $\tau$ of pheromone in that path. On the other hand, ants that chose path B are still in the middle of the course. When ants that chose path $\mathrm{A}$ in the first travel are coming back to the nest, three of them choose path $\mathrm{A}$ and one chooses path B because of the pheromone level. For $t=2$, ants that chose path A (in $t=1$ ) are now in the nest and they have increased the pheromone level in this path, whilst the ant that chose path B (in $t=1$ ) instead path $\mathrm{A}$, to come back to the nest, is still in the middle of this path. The ants that chose path $\mathrm{B}$ in $t=0$ are just arriving to the food source. When they return to the nest, due to the greater pheromone concentration, three choose path A and only one chooses path B. Finally for $t=3$, there are seven ants crossing the path A and only one the path B. For later times, path A will have a greater probability to be chosen than path $\mathrm{B}$, since the pheromone concentration decreases in path $\mathrm{B}$ (due to the fact that it is not used) and increases in path A. However, there are always some ants exploring new paths. The later allow to ants no stagnate only in one path, giving to the optimization technique its global nature.

The process described above shows how the ants are led for the shortest path between the nest and the food source. This communication process is called artificial stigmergic [9].

\section{ACO Applied to Microwave Corrugated Filter Design}

3.1. Problem Description. The ACO method is applied in this paper to the design of $\mathrm{H}$-plane cavity corrugated filters. Since the structure is invariant in height, the filter can be considered as the cascade association of a set of cavities of length $D_{i}$ coupled through centred irises of width $W_{j}$, as it is shown in Figure 2. The design consists in finding the set $D_{i}$ and $W_{j}$ letting to an $S_{21}$ parameter (or transmission parameter) that matches a given goal transfer function satisfying a specified error criteria. Because the ACO method is in essence discrete, it is necessary to use a discrete search space. This fact is commented in some detail later on (namely, in Section 4).

For a filter of order $n, n+2$ cavity segments and $n+1$ iris apertures are necessary as Figure 2 shows and thus the total number of elements to be found is $n t=2 n+3$.

These filters are widely used for high frequency $(\mathrm{GHz})$ and high power communication applications (e.g., satellite communications or radar) and consist of a metallic rectangular waveguide with rectangular irises along it (see [18-21] for more details).

3.2. ACO Algorithm Implementation. In this work, a modified version of the ACO algorithm described in [17] is developed, taking into account those differences between the microwave filter design problem and the problems (i.e., the Travelling Salesman Problem-TSP) dealt with in that reference; for example, in [17] once an ant has crossed one node (city), due the nature of the problem, this node is partially removed from the search space (the ant has to be only once in each node). In the application handled in this work, once an ant has selected one node (filter element) it remains in the search space, increasing in this way the complexity of the problem.

For the current application, a colony of $N$ ants is used to find the best solution (a combination of $2 n+3$ filter elements) in a search space of dimension $M$. The value of $M$ is not relevant at all, but for the computational effort. In Section 4, it will be described how to choose $M$ taking into account the size of the filter and the required mechanical tolerance. The elements are chosen depending on their pheromone concentration and some heuristic information (the a priori preference that any possible element has to be used in a filter position). Each ant represents a solution consisting in an $n t \times 1$ array, where each entry represents one chosen element in the search space (namely, $D_{i}$ or $W_{j}$, as defined in Figure 2).

The algorithm described in [17] consists of several steps: parameter initialization, pseudorandom proportional rule, local pheromone update, evaluation of the partial solutions by means of a fitness function, global pheromone update, and finally, heuristic information update as it is described in Figure 3. For the method presented in this paper, the global pheromone update and heuristic information update procedures have been modified regarding those presented in [17].

The algorithm starts by initializing some specific variables and the initial point, which is randomly selected. Then, each 


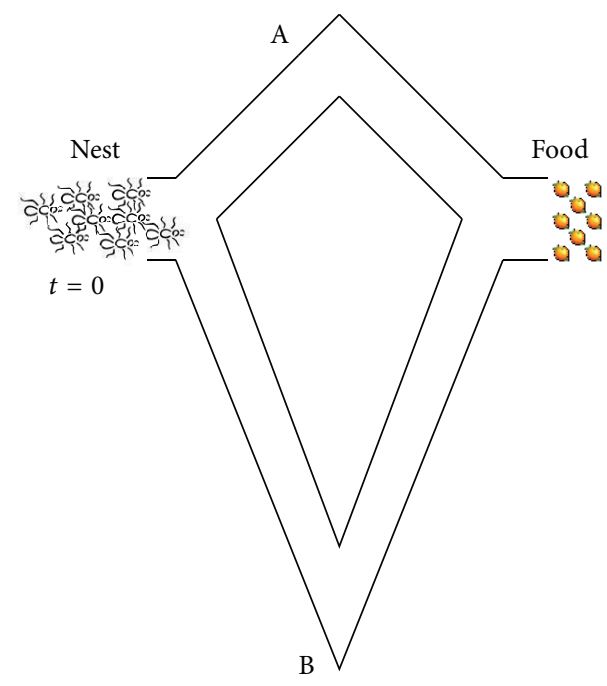

(a)

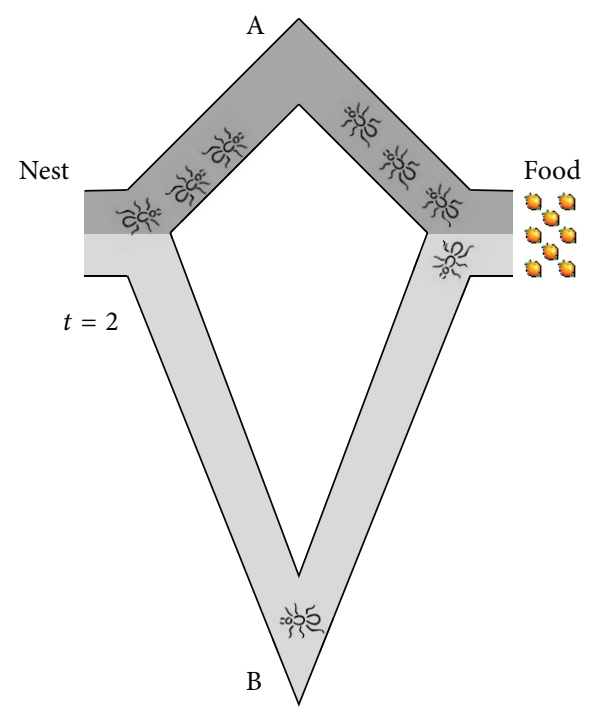

(c)

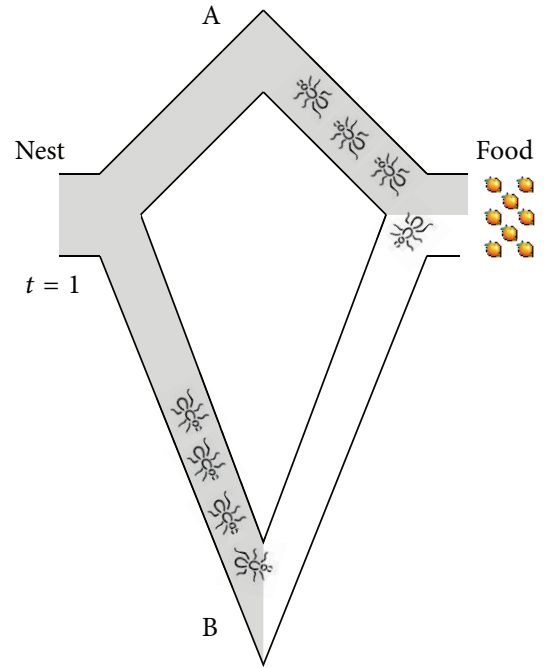

(b)

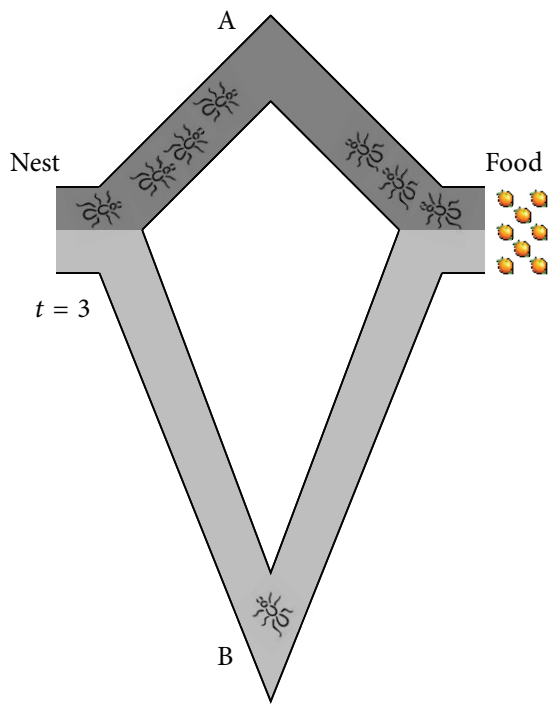

(d)

Figure 1: Ant colony behaviour.

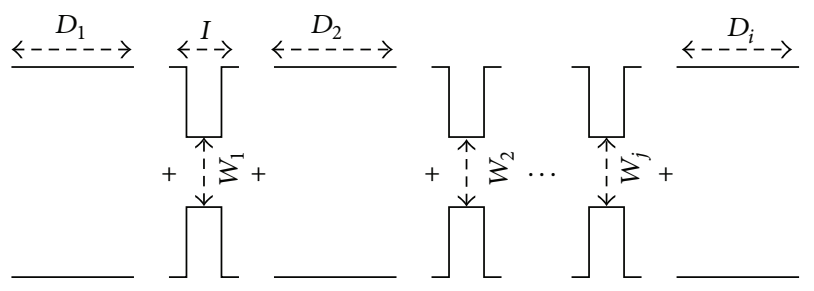

FIGURE 2: Number of iris segments and iris apertures to be obtained.

ant selects one element (for every filter position) based on a probabilistic decision criteria (the elements for a filter are numbered from 1 to $n t$ ). Since each ant selects an element for each filter position, a local pheromone update is performed for this element. This process is repeated until all the ants have selected all the elements for the sets $D_{i}$ and $W_{j}$. After this step, the electromagnetic problem is solved (the transmission parameter is obtained) and the partial solutions found by the ants are scored. The score (Error) of every partial solution is obtained by using a fitness function. Finally, a global pheromone and heuristic information updates are performed. In the following the modified steps (i.e., the global pheromone and heuristic information update steps) are fully described.

The probabilistic criterion decision used herein is called pseudorandom-proportional rule $[9,17]$ and it can be described as follows. Let the variable $q_{0} \in[0,1]$ be an initial and fixed parameter of the algorithm and $q$ a random value such that $q \in[0,1]$; the probability $p_{i, j}^{k}(t)$ that some $j$ th 


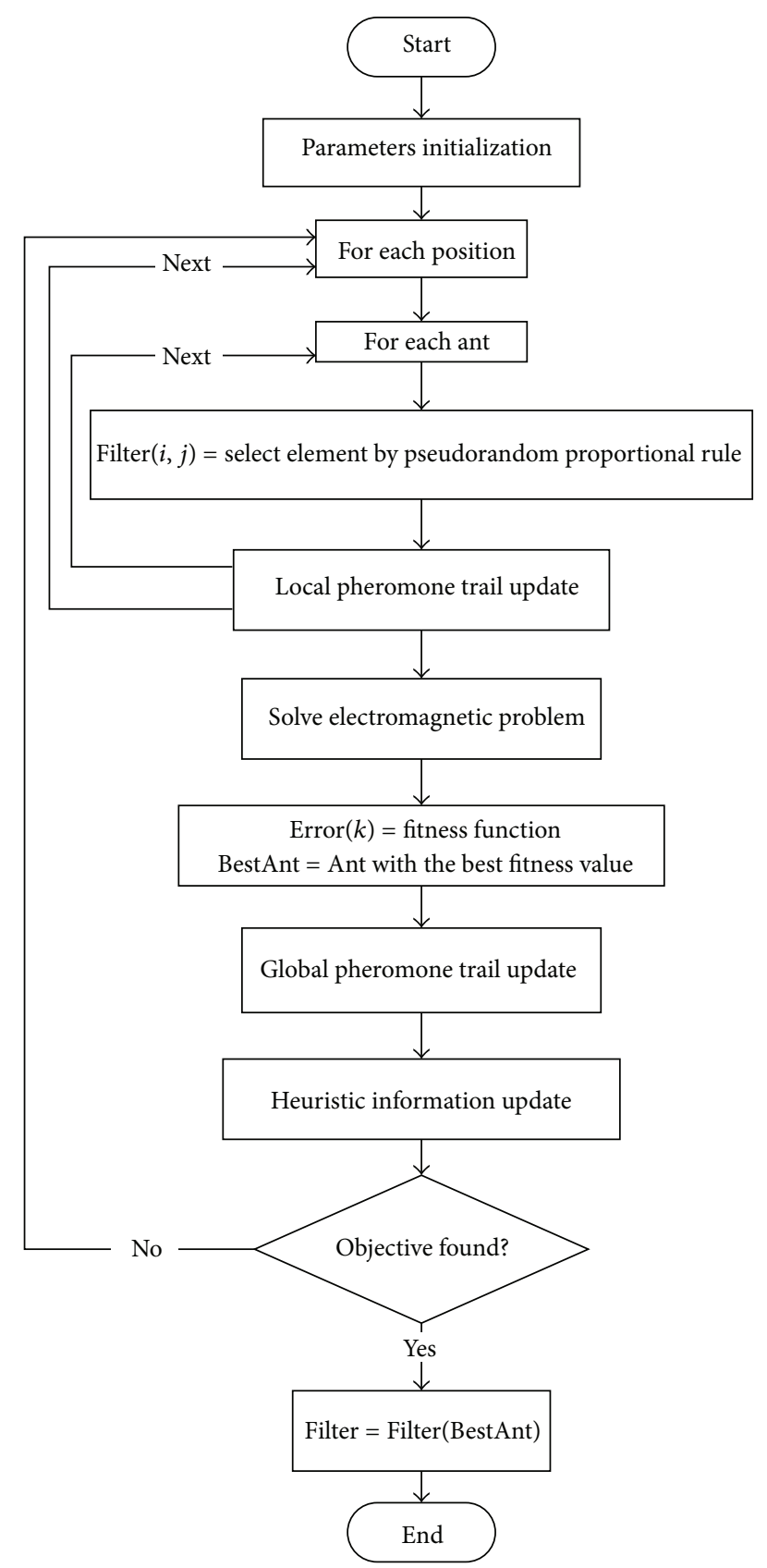

FIGURE 3: ACO algorithm flowchart.

element has to be chosen for the $i$ th filter position by the $k$ th ant, at some instant $t$, is defined as follows:

$$
\begin{gathered}
\text { if } q \leq q_{0}, \quad p_{i, j}^{k}= \begin{cases}1 & \text { if } j=\arg \max _{j \in I_{i}}\left\{\tau_{i, j}\left(\eta_{i, j}^{k}\right)^{\beta}\right\} \\
0 & \text { otherwise, }\end{cases} \\
\text { if } q>q_{0}, \quad p_{i, j}^{k}=\frac{\tau_{i, j}\left(\eta_{i, j}^{k}\right)^{\beta}}{\sum_{j \in J_{i}} \tau_{i, j}\left(\eta_{i, j}^{k}\right)^{\beta}},
\end{gathered}
$$

where $\tau_{i, j}$ is the pheromone concentration of the $j$ th element when it is used for the $i$ th filter position and $\eta_{i, j}^{k}$ is the heuristic information that scores the a priori preference of a $j$ th element to be chosen by the $k$ th ant for the $i$ th filter position. The parameter $\beta$ scores the influence of the heuristic information on the pseudorandom-proportional rule and $J_{i}$ is the set of available elements for a specific $i$ th filter position.

Pheromone concentration controls the relevance of an element of the search space on the obtained response when it is used in one filter position (i.e., in a given entry of the ant array). In $\mathrm{ACO}$ algorithm two kinds of pheromone update are used: the local pheromone update and the global pheromone update. The local pheromone update is performed by $[9,17]$ :

$$
\tau_{i, j}=(1-\varphi) \tau_{i, j}+\varphi \tau_{0},
$$

where $\tau_{0}$ is the initial amount of pheromone concentration and $\varphi$ is the local evaporation coefficient.

The global pheromone is updated for the $j$ th element in the search space when it is chosen by the best ant at time $t$ and then introduced at the $i$ th position in the corresponding best solution array. The functions used in [17] to implement the pheromone update take into account only the fitness function value at time $t$. In this work, a modified fitness function involving the values at times $t$ and $t-1$ is proposed. This modification helps the algorithm to give more importance to those elements belonging to solutions that have improved the design regarding the previous one. It is shown in the following

$$
\begin{gathered}
\tau_{i, j}(t+1)=(1-\rho) \tau_{i, j}(t)+\Delta \tau_{i, j}(t), \\
\Delta \tau_{i, j}(t)=Q \frac{\text { Error }_{t}^{\text {best }}}{\text { Error }_{t-1}^{\text {best }}},
\end{gathered}
$$

where parameter $\rho$ is the global evaporation coefficient, $Q$ is a fixed value that controls the order of magnitude of the pheromone increment, and Error ${ }^{\text {best }}$ is the best fitness function value found by the colony. The fitness function is basically given by the mean of the standard deviation values between each point in frequency of the goal transfer function and their corresponding in the transfer function found by an ant. It is expressed, for the $k$ th ant, as follows:

$$
\text { Error }^{k}=\frac{1}{n f} \sum_{i=1}^{n f} \sqrt{\left(\left|S_{21}^{k}(i)\right|-m_{i}\right)^{2}+\left(\left|S_{21}^{\mathrm{obj}}(i)\right|-m_{i}\right)^{2}},
$$

where $n f$ is the number of frequency points, $S_{21}^{k}$ is the filter response given by the partial solution found by the $k$ th ant, $S_{21}^{\mathrm{obj}}$ is the goal filter response, and $m_{i}=\left(S_{21}^{k}(i)+S_{21}^{\mathrm{obj}}(i)\right) / 2$. The efficiency of the ACO method mainly depends on the choice of the fitness function. If the value calculated by this function is not an accurate representation of the actual situation, ants easily will be in wrong search space zones, that is, in zones with values that do not minimize (4).

Finally, the heuristic information scores the a priori preference of using one element in one filter position. For the problems dealt with in [17], an a priori evaluation of ethe problem, taking into account all the possibilities is used to calculate a heuristic value. This is possible because the nature of those problems makes it computationally feasible. 
However, this strategy is not applicable to the electromagnetic problems considered here (microwave corrugated filters) due to its computational cost. On the other hand, there are no criteria to know, a priori, whether some elements of the search space are suitable or not for the filter in a given position. For this reason, in this work a new and practical strategy to calculate the heuristic information is proposed. This strategy is based on the improvement directions of each ant regarding the best solution found by the colony. Figure 4 shows the flowchart describing this innovative approach.

In Figure 4 the parameter $\eta^{k}$ stands for the heuristic information that has the $k$ th ant, Filter ${ }_{t}^{k}$ is the filter found by the $k$ th ant in time $t$, Filter ${ }^{\text {best }}$ is the best filter found by the colony until time $t$, and $U$ is a $1 \times(u b-l b+1)$ random array with uniform probability distribution. This rule suggests that those elements of the search space lying between the value of a given entry of the filter vector found in time $t$ by the $k$ th ant and the value of the same entry of the best found filter will have a greater preference than the rest of the possible elements.

Finally, the algorithm parameters directly depend on the problem to be solved and it is very common to use an empirical selection $[9,10,17]$.

The heuristic information update, developed in this work, avoids the electromagnetic problem evaluation for every ant and iteration or for all the search space components as it is proposed in other works (e.g., $[10,12,17])$. This also allows an important reduction of the computational effort. This aspect is particularly useful for electromagnetic problems where heavy numerical algorithms must be run to solve the physical phenomenon.

\section{Results}

The optimization technique developed in this paper has been applied to the design of a microwave rectangular waveguide high-pass filter at C-band $(4-10 \mathrm{GHz})$ and of a band-pass filter at X-band (10.7-11.3 GHz). The $S_{21}$ parameter is obtained and compared with that obtained by the classical Genetic Algorithm (GA). The GA Matlab toolbox has been used for this task. Also the average convergence performance for ACO is analyzed and compared with GA by means of Monte Carlo simulations.

The discretization step used for $D_{i}$ and $W_{j}$ is $0.1 \mathrm{~mm}$ (which is less than a hundredth of the wavelength at the operating frequencies). This value, along with the physical filter dimensions, yields the search space dimension, $M$. Nevertheless, this method is also valid for any discretization step; this value only affects the desired design accuracy and computation time. The electromagnetic problem has been solved using a Mode-Matching (MM) method tool [22].

The main parameters for the ACO algorithm are $\beta=2$, $\rho=0.1, Q=10, q_{0}=0.7$, and $\varphi=0.1$. Additionally, for both optimization algorithms, the same number of artificial agents (ants for ACO and individuals for GA) has been used (10 agents) in order to make a fair comparison. The initial population is randomly established and it is set equal for both methods. For convenience and to illustrate, in some way, the

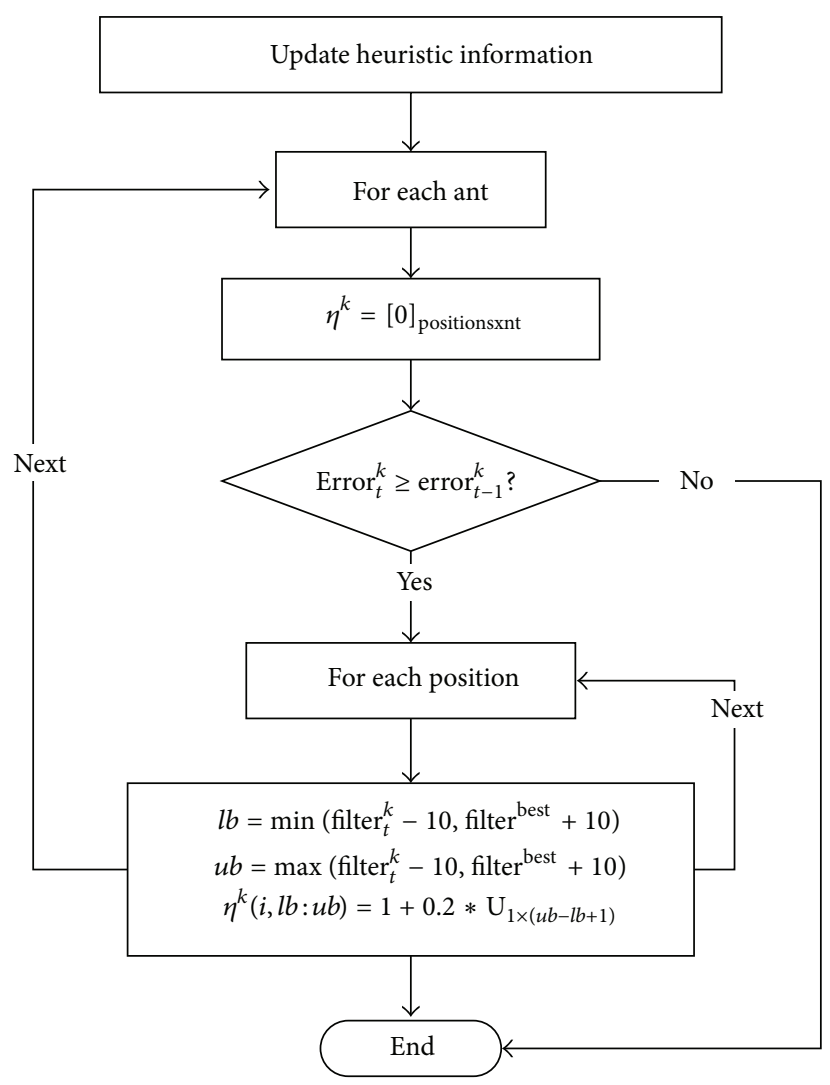

FIgURE 4: Heuristic information update.

quality of the results, the filter response due to the initial population is also shown along with those obtained by ACO and GA (see Figures 5 and 7). Moreover, also the same fitness function (4) is used for ACO and GA. The simulations are performed in a desktop computer of $2.2 \mathrm{GHz}$ of processor and $4 \mathrm{GHz}$ of Random Access Memory (RAM).

On the other hand, the main parameters used for GA are elite count equal to 2, crossover fraction equal to 0.01 , crossover function intermediate, mutation function uniform, and mutation rate equal to 0.01 .

As it is commented in $[23,24]$ the comparison of this kind of algorithms is not evident nor trivial. However, the aim in this work is to demonstrate the validity of the proposed algorithm rather than to demonstrate whether it is better or not than other ones; therefore, a more practical strategy to do this comparison is used and the guidelines proposed in $[23,24]$ are avoided.

4.1. High-Pass Filter. The filter has been designed in a WR-137 $(34.85 \times 15.8 \mathrm{~mm})$ waveguide. For this filter the defined search space for iris apertures is $4-34.8 \mathrm{~mm}$ and $5-20 \mathrm{~mm}$ for iris segments. The parameter $I$ in Figure 2 is set to $0.5 \mathrm{~mm}$. The order of this filter is 6 with a cut-off frequency of $5.4 \mathrm{GHz}$. The goal $S_{21}$ parameter has been taken from [18] and for convenience is shown in Figure 5.

The dimensions of fifteen filter elements have to be calculated for this filter. However, as it is well known [4, 18, 


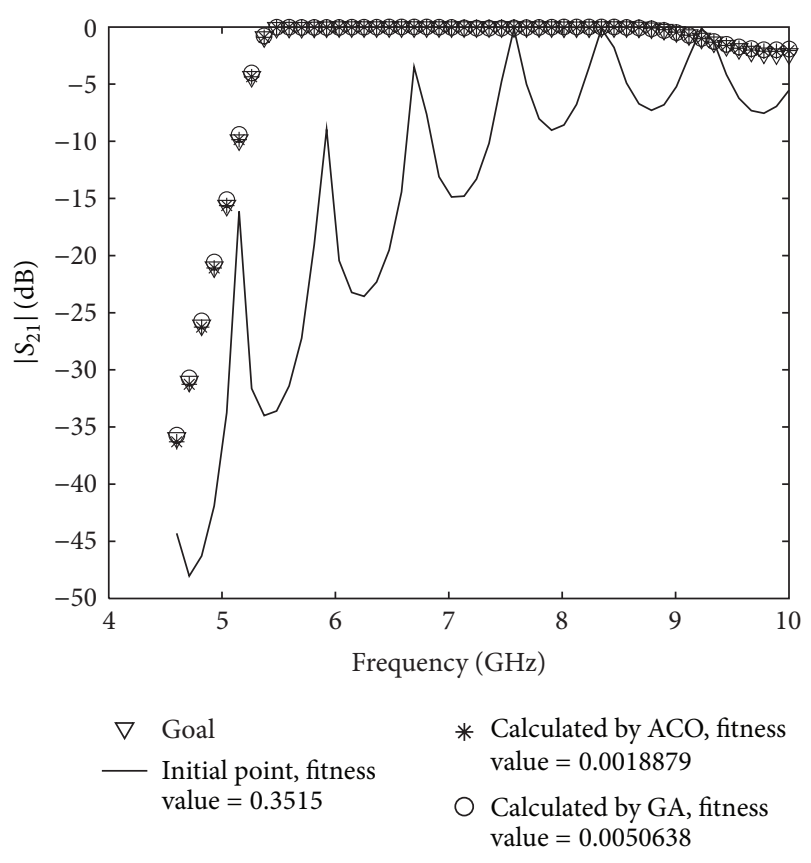

FIGURE 5: High-pass filter response.

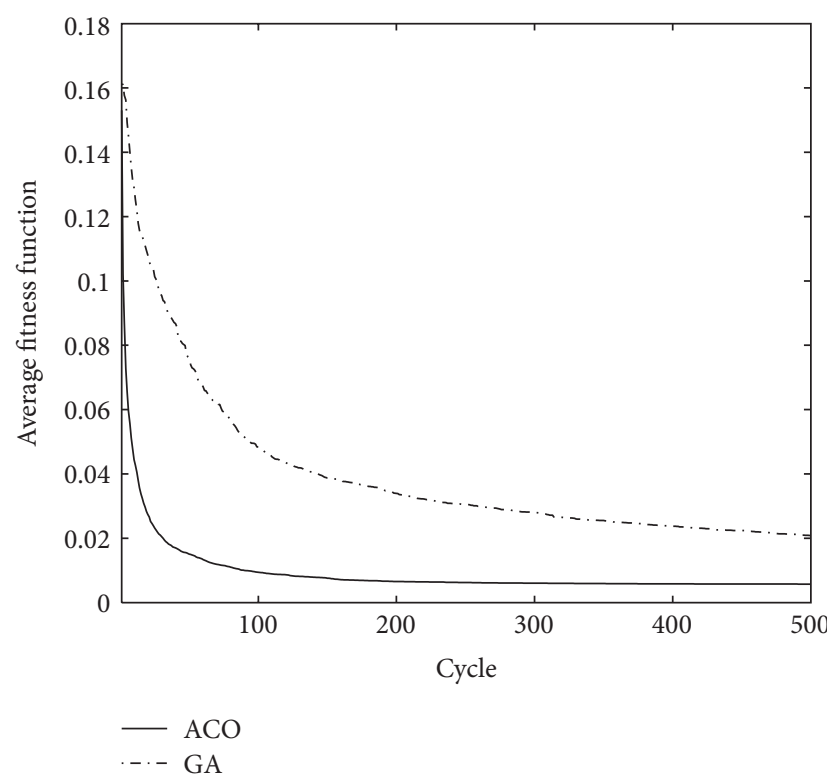

Figure 6: Average convergence performance for high-pass filter.

20], a typical waveguide filter design is a symmetric structure. This property is used in this simulation; thus, only a total of eight element dimensions have been calculated.

Figure 5 shows the responses for the designs found by ACO and GA methods. Both methods have found very accurate designs. However, for the same number of cycles (500) the ACO method has obtained the best fitness function value $(0.0018879)$. On the other hand, Figure 6 shows the average convergence performance obtained for both ACO and GA methods by means of Monte Carlo simulations (100

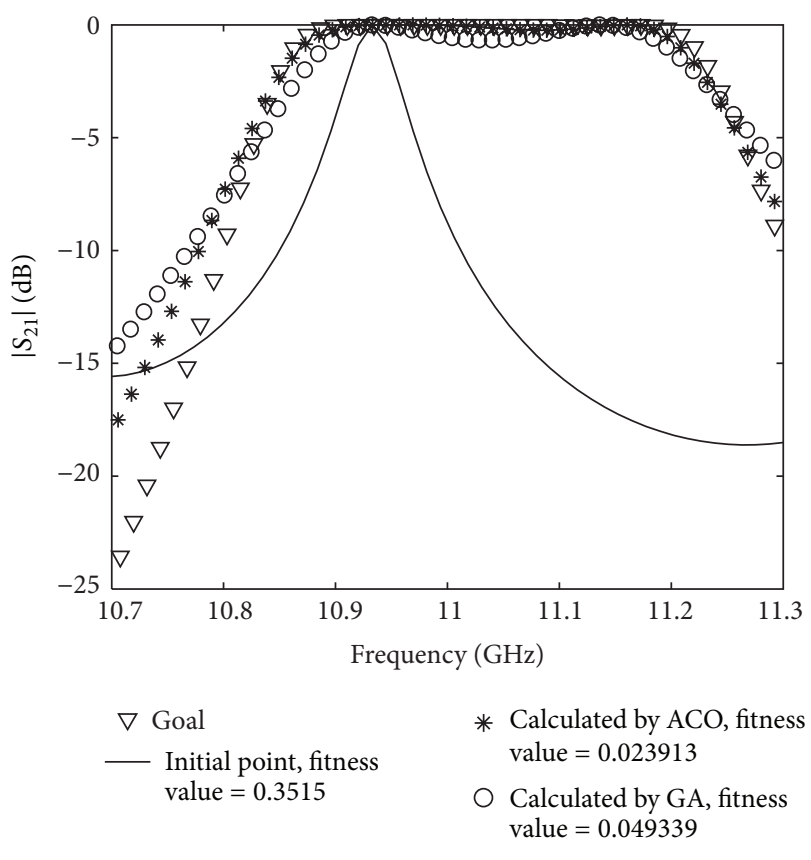

FIgURE 7: Band-pass filter response.

trials). In this figure, the mean of the fitness values, for each point in frequency, of the solution in each trial is shown. From this figure it can be observed that the ACO method reaches more accurate designs faster than the used GA method. In practical terms, the ACO method finds a good design after 100 cycles whilst GA needs at least 400 cycles.

Regarding to the processing time per cycle, the same amount of time has been found for both methods. Firstly, it is because they use the same number of artificial agents (10 agents), and secondly, because in this kind of problems that processing time is dominated by the solution of the fitness function (i.e., the solution of an electromagnetic problem that is the order of 1-3 seconds) rather than the implementation of the optimization algorithm itself. Thus, neglecting the time for solving the fitness function, which is not directly part of an optimization algorithm, the processing time per cycle for both ACO and GA was found of the order of hundred of microseconds. Finally, regarding the memory consumption any rigorous analysis was performed but it is important to emphasize that with a desktop computer like the one described in Section 4 any memory overload was found for any of the tested algorithms. Therefore, under these conditions, the most equitable parameter to measure the algorithms efficiency is the number of required cycles to obtain a suitable solution.

4.2. Band-Pass Filter. This filter has been designed in a WR$75(19.05 \times 9.525 \mathrm{~mm})$ waveguide. For this filter, the defined search space for iris apertures was $3-19 \mathrm{~mm}$ and $10-20 \mathrm{~mm}$ for iris segments. The parameter $I$ in Figure 2 is set to $2 \mathrm{~mm}$. The order of this filter is 4 , with a central frequency of $11 \mathrm{GHz}$ and a bandwidth of $300 \mathrm{MHz}$. The goal $S_{21}$ parameter is a 


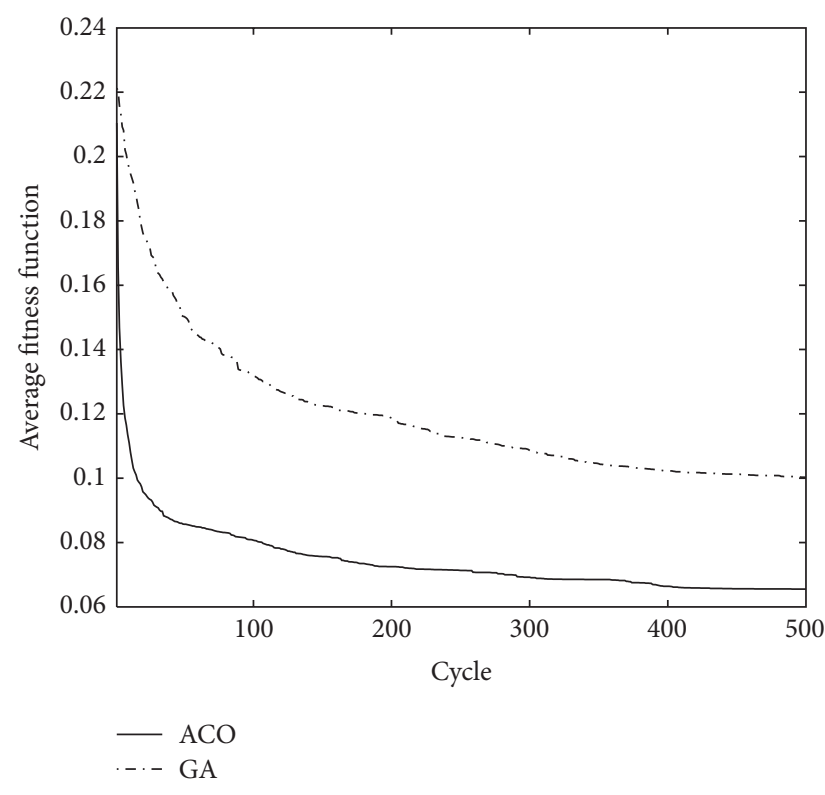

FIGURE 8: Average convergence performance for band-pass filter.

standard four-pole Chebyshev curve and for convenience is shown in Figure 7.

For this filter the dimensions of eleven filter elements have to be calculated. The symmetry property is also used for this simulation; thus, only six elements dimensions have been calculated.

Figure 7 shows the responses for the designs found by ACO and GA methods. It is evident that the ACO design is closer to the goal than the one found by GA. In this example, the ACO method gives up to the double of the accuracy regarding the GA response as it can be observed in the fitness values in the legend of Figure 7.

On the other hand, Figure 8 shows the average convergence performance obtained for both the ACO and the GA methods by means of Monte Carlo simulations (100 trials). It can be seen that the ACO method also for this design obtains a much faster convergence than the GA method, that is, a better fitness value in fewer cycles.

As it was commented in Section 1, the design of a filter generally requires two optimization steps, one for a global search and another one (subsequent) for a local search. The results shown in this work can be significantly improved by using a local search algorithm; however, it is not the aim of this work to implement a second search step, but to demonstrate the usefulness of the proposed ACO algorithm to design microwave corrugated filters.

\section{Conclusions}

A new, practical, and useful application of the Ant Colony Optimization (ACO) method for the design of microwave waveguide filters has been presented. The modifications of the ACO algorithm, to adapt it for microwave filter designs, were also fully described.
The ACO method used here has been validated with two examples: a high-pass filter at C-band and a band-pass filter in X-band. Accurate results have been reached comparable or better than those obtained by the well-established Genetic Algorithms (GA) for both examples. Moreover, it was found that ACO method achieved a much faster average convergence than GA also for both examples.

A practical and useful strategy to update the heuristic information, which avoids the previous evaluation of the electromagnetic problem, has been developed and described.

The results obtained with this ACO method can be used as final designs (in some cases) or as a very good starting point for a local optimization stage of a design procedure. Also this ACO method can be used along with other electromagnetic simulators in order to solve other kinds of problems, for example, as that solved in [4].

\section{Acknowledgment}

This research work has been partially supported by the Spanish Ministry of Education with a FPU Grant (AP200803300).

\section{References}

[1] D. Swanson and G. Macchiarella, "Microwave filter design by synthesis and optimization," IEEE Microwave Magazine, vol. 8, no. 2, pp. 55-69, 2007.

[2] M. F. J. Nogales, J. P. García, J. Hinojosa, and A. Alvarez-Melcón, "Genetic algoritms applied to microwave filters optimization and design," in Progress in Electromagnetics Research Symposium, pp. 99-103, Cambridge, Mass, USA, July 2008.

[3] D. Budimir and G. Goussetis, "Design of asymmetrical RF and microwave bandpass filters by computer optimization," IEEE Transactions on Microwave Theory and Techniques, vol. 51, no. 4, pp. 1174-1178, 2003.

[4] J. V. M. Ros, P. S. Pacheco, H. E. González et al., "Fast automated design of waveguide filters using aggressive space mapping with a new segmentation strategy and a hybrid optimization algorithm," IEEE Transactions on Microwave Theory and Techniques, vol. 53, no. 4, pp. 1130-1141, 2005.

[5] J. Robinson and Y. Rahmat-Samii, "Particle swarm optimization in electromagnetics," IEEE Transactions on Antennas and Propagation, vol. 52, no. 2, pp. 397-407, 2004.

[6] Y. Rahmat-Samii and E. Michielssen, Electromagnetic Optimization by Genetic Algorithms, Wiley-Interscience, New York, NY, USA, 1999.

[7] J. Nelder and R. Mead, "A simplex method for function minimization," Computer Journal, vol. 7, pp. 308-313, 1965.

[8] M. Dorigo, V. Maniezzo, and A. Colorni, "Ant system: optimization by a colony of cooperating agents," IEEE Transactions on Systems, Man, and Cybernetics, Part B: Cybernetics, vol. 26, no. 1, pp. 29-41, 1996.

[9] M. Dorigo and T. Stützle, Ant Colony Optimization, MIT Press, Cambridge, Mass, USA, 2004.

[10] E. Rajo-lglesias and Ó. Quevedo-Teruel, "Linear array synthesis using an ant-colony-optimization-based algorithm," IEEE Antennas and Propagation Magazine, vol. 49, no. 2, pp. 70-79, 2007. 
[11] A. Akdagli, K. Güney, and D. Karaboga, "Pattern nulling of linear antenna arrays by controlling only the element positions with the use of improved touring ant colony optimization algorithm," Journal of Electromagnetic Waves and Applications, vol. 16, no. 10, pp. 1423-1441, 2002.

[12] Ó. Quevedo-Teruel and E. Rajo-Iglesias, "Ant colony optimization in thinned array synthesis with minimum sidelobe level," IEEE Antennas and Wireless Propagation Letters, vol. 5, no. 1, pp. 349-352, 2006.

[13] P. Rocca, L. Manica, and A. Massa, "An improved excitation matching method based on an ant colony optimization for suboptimal-free clustering in sum-difference compromise synthesis," IEEE Transactions on Antennas and Propagation, vol. 57, no. 8, pp. 2297-2306, 2009.

[14] L. Chang, C. Liao, W. B. Lin, L.-L. Chen, and X. Zheng, "A hybrid method based on differential evolution and continuous ant colony optimization and its application on wideband antenna design," Progress in Electromagnetics Research, vol. 122, pp. 105-118, 2012.

[15] M. Mussetta, F. Grimaccia, and R. E. Zich, "Comparison of different optimization techniques in the design of electromagnetic devices," in Proceedings of IEEE World Congress on Computational Intelligence (WCCI '12), Brisbane, Australia, June 2012.

[16] O. Quevedo-Teruel, E. Rajo-Iglesias, and M. Ng Mou Kehn, "Numerical and experimental studies of split ring resonators loaded on the sidewalls of rectangular waveguides," IET Microwaves, Antennas and Propagation, vol. 3, no. 8, pp. 12621270, 2009.

[17] M. Dorigo and L. M. Gambardella, "Ant colony system: a cooperative learning approach to the traveling salesman problem," IEEE Transactions on Evolutionary Computation, vol. 1, no. 1, pp. 53-66, 1997.

[18] G. Matthaei, L. Young, and E. M. T. Jones, Microwave Filters, Impedance-Matching Networks, and Coupling Structures, Artech House, Dedham, Mass, USA, 1980.

[19] N. Marcuvitz, Waveguide Handbook, P. Peregrinus, London, UK, 1993.

[20] R. Levy, R. V. Snyder, and G. Matthaei, "Design of microwave filters," IEEE Transactions on Microwave Theory and Techniques, vol. 50, no. 3, pp. 783-793, 2002.

[21] P. Soto, V. E. Boria, J. M. Catalá-Civera, N. Chouaib, and M. Guglielmi, "Analysis, design and experimental verification of microwave filters for safety issues in open-ended waveguide systems," IEEE Transactions on Microwave Theory and Techniques, vol. 48, no. 1, pp. 2133-2140, 2000.

[22] A. Díaz-Morcillo, I. A. Mantilla-Gaviria, and J. V. BalbastreTejedor, "A mode-matching/finite element hybrid strategy for analizing waveguide discontinuities and resonant cavities," in Progress In Electromagnetics Research Symposium (PIERS '08), Cambridge, Mass, USA, July 2008.

[23] D. H. Wolpert and W. G. Macready, "No free lunch theorems for optimization," IEEE Transactions on Evolutionary Computation, vol. 1, no. 1, pp. 67-82, 1997.

[24] Ó. Quevedo-Teruel, E. Rajo-Iglesias, and A. Oropesa-García, "Hybrid algorithms for electromagnetic problems and the nofree-lunch framework," IEEE Transactions on Antennas and Propagation, vol. 55, no. 3, pp. 742-749, 2007. 

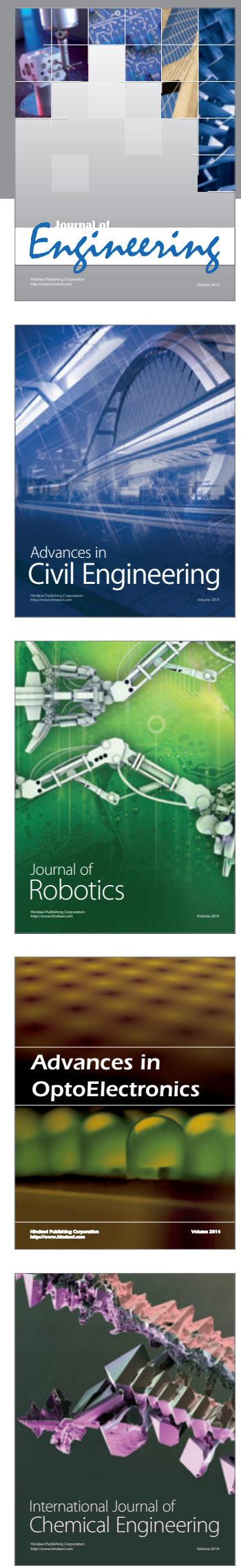

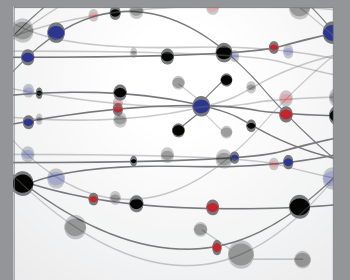

The Scientific World Journal
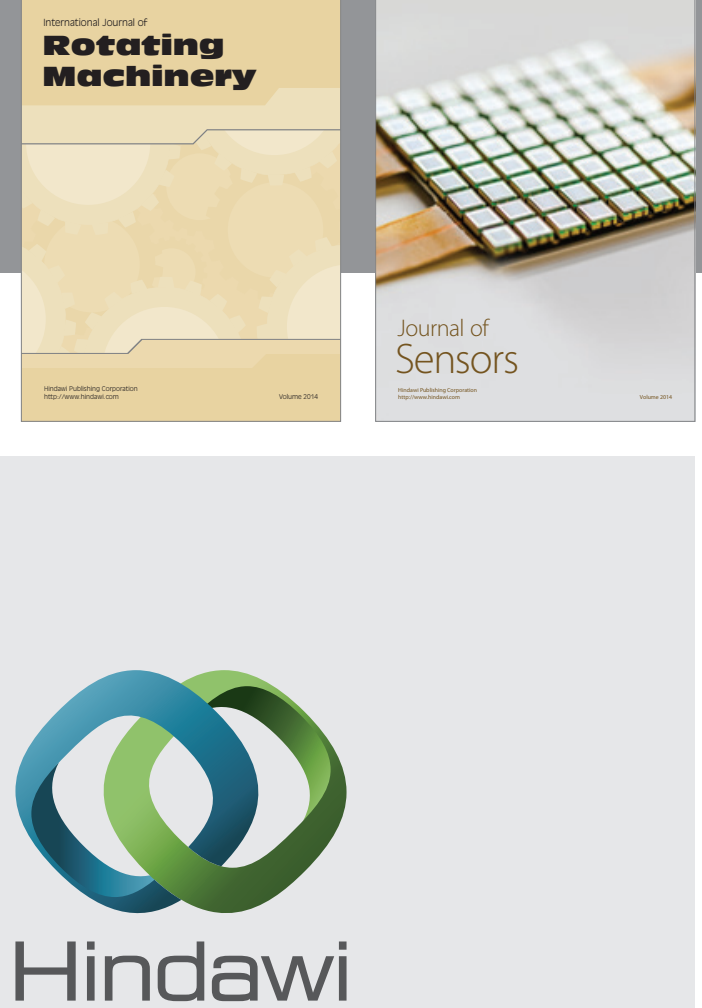

Submit your manuscripts at http://www.hindawi.com
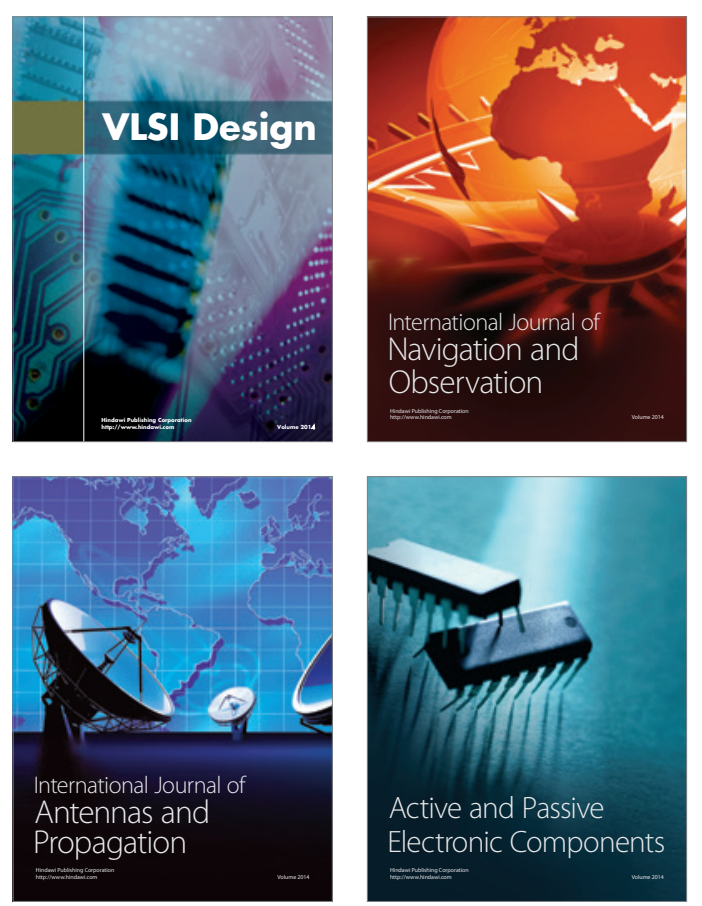
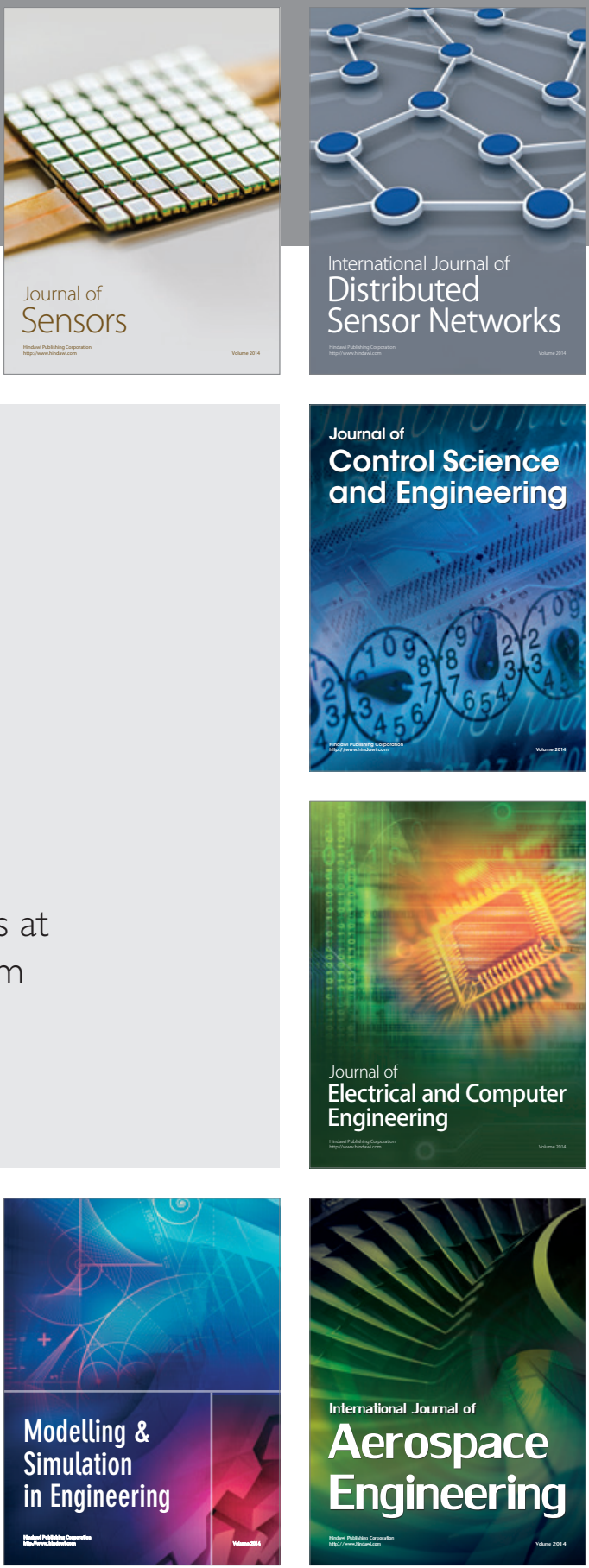

Journal of

Control Science

and Engineering
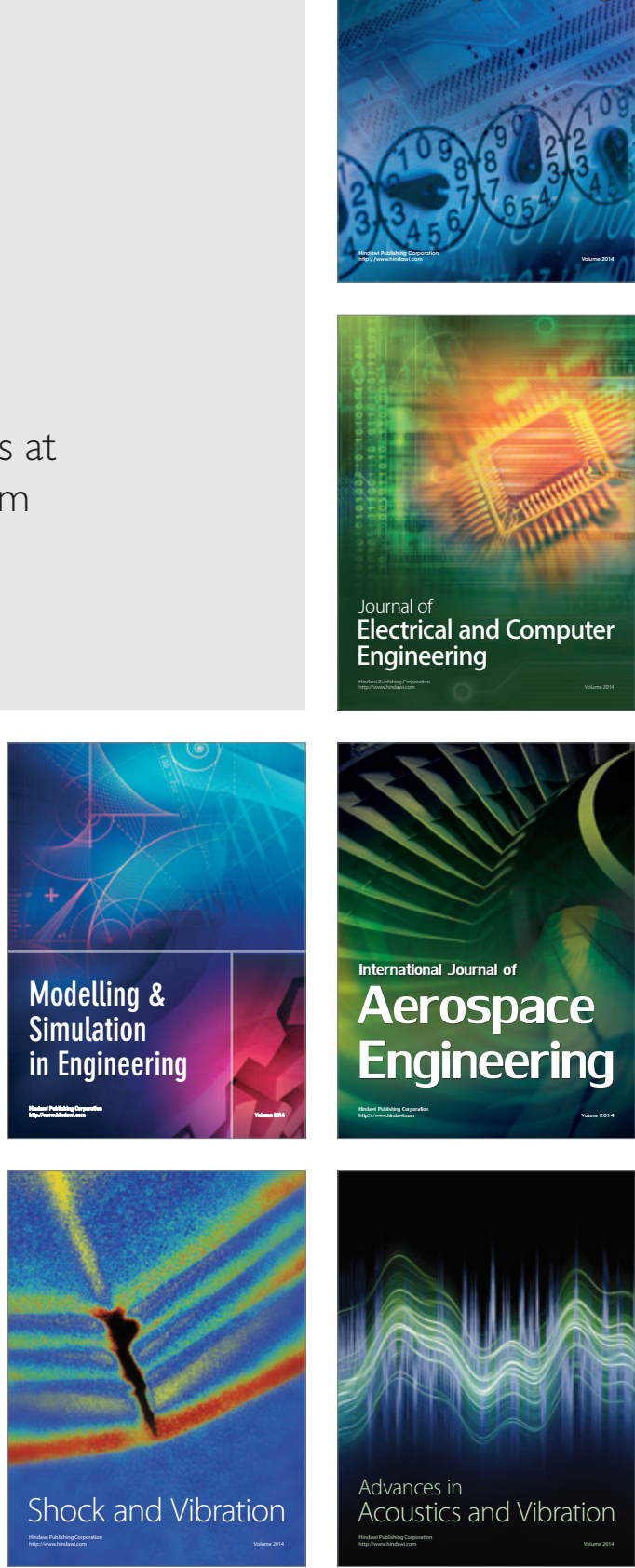